\title{
A STUDY OF RURL GIRLS EXTENSION PROGRAMS EFFECT IN TRANSFERRING EXTENSION RECOMMENDATIONS TO RURAL FAMILIES IN THE FIELD OF HOME ECONOMICS "A FIELD STUDY IN RURAL DAKAHLIA GOVERNORATE".
}

El-Gamal, M.M.A.; M.A.M. Abd El-Magieed and Zozo F.E. Sallam Agricultural Extension and Rural Society Dept., Faculty of Agriculture, University of Mansoura.

دراسة لأثر البرامج الإرشادية للفتيات الريفيات فى نقل التوصيات الإرشـادية للأسر الريفية

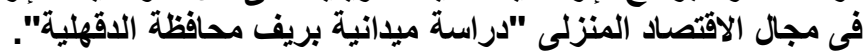

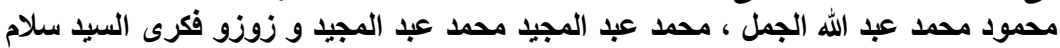
قسم الإرشاد الزراعى والمجتمع الريفى ـ كلية الزراعة ـ جامعة المنصورة.

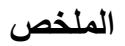

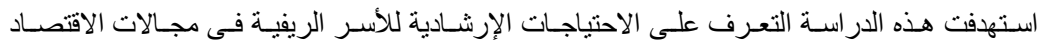

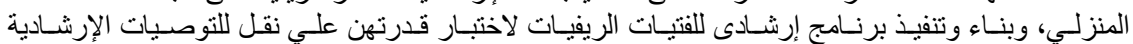

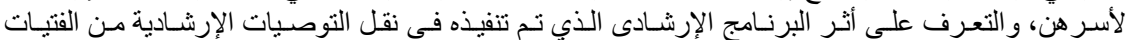
الريفيات للأسر الريفية فى المجالات الأكثر الاتنياجا.

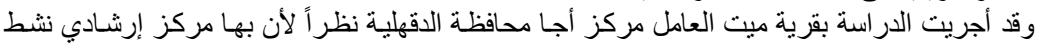

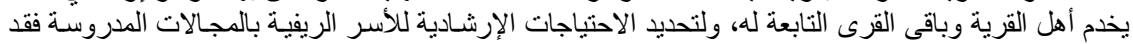

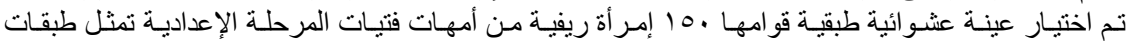

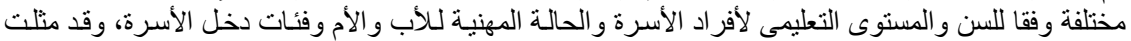

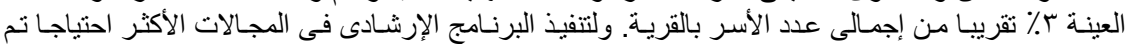

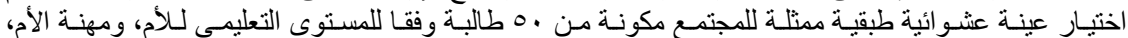

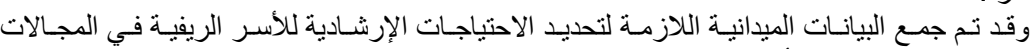

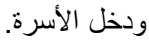

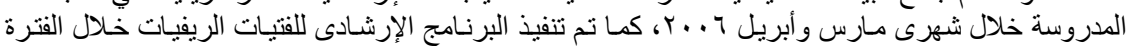

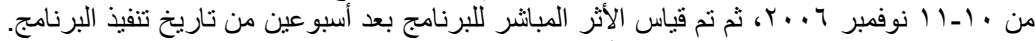

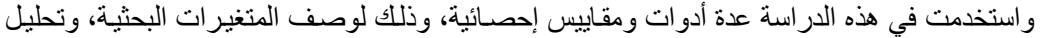

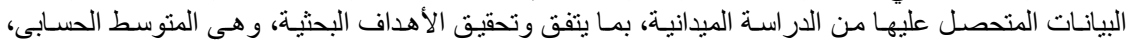
واختبار (t).

$$
\text { وقد نوصلت الدراسة لعدد من النتائج من أهمها: }
$$

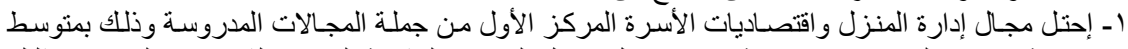

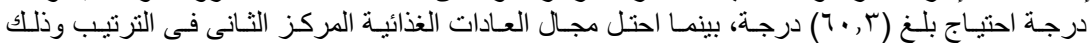

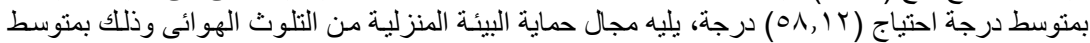

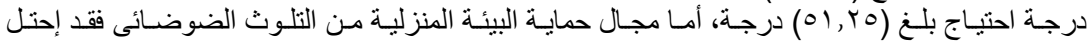

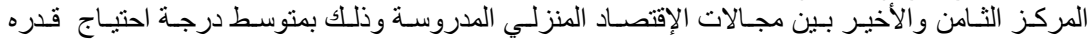

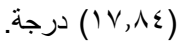

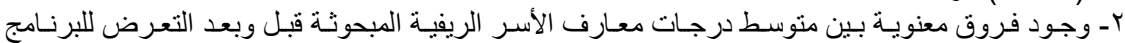

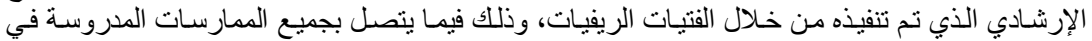

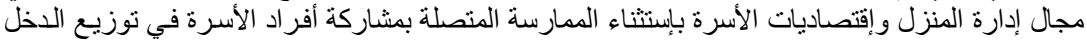

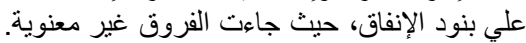

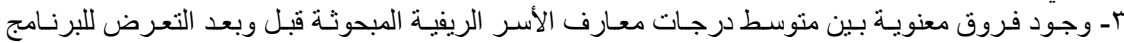

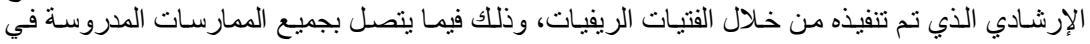




\section{El-Gamal, M.M.A. et al.}

مجال العادات الغذائية بإنتثناء الممارسة المتصلة بتناول الخبز الأبيض دون السن أو البلدي، حيث جاءت

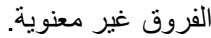

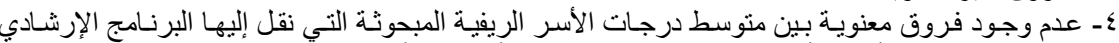

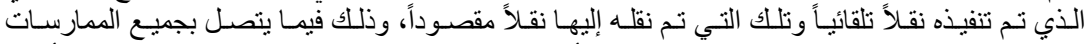

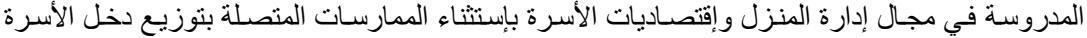

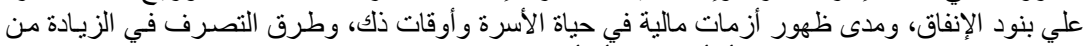

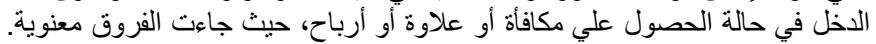

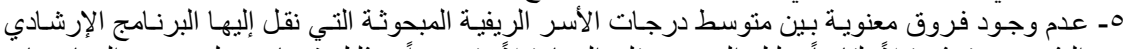

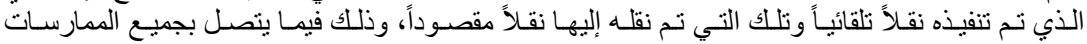

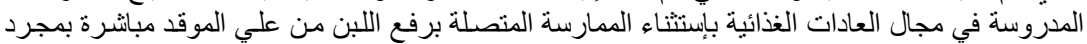

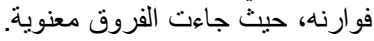

\section{المقدمة}

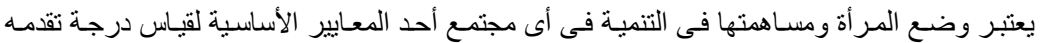

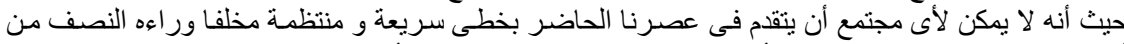

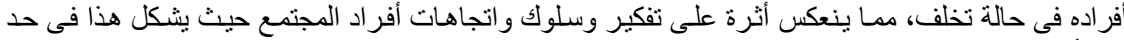

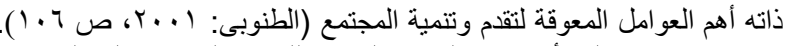

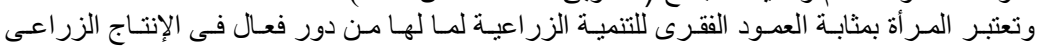

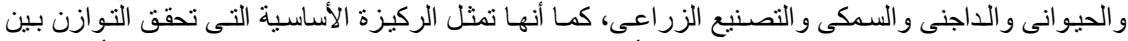

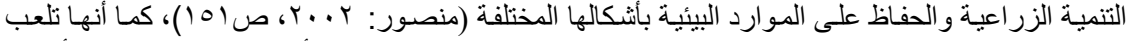

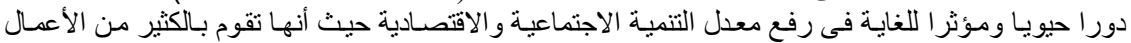

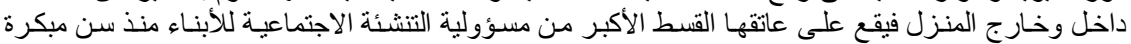

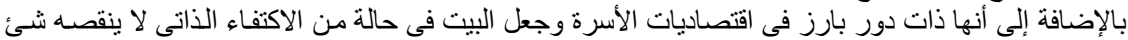

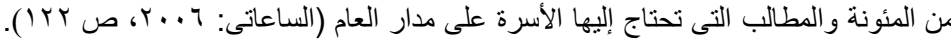

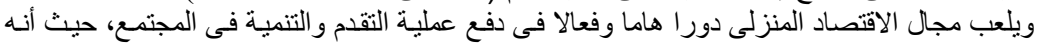

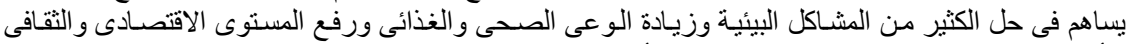

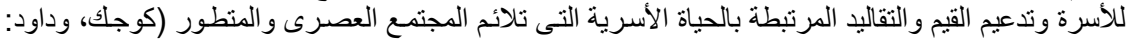

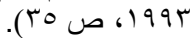

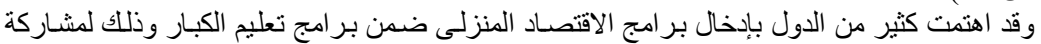

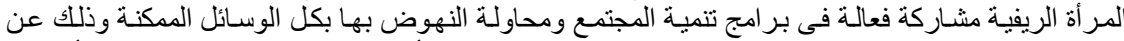

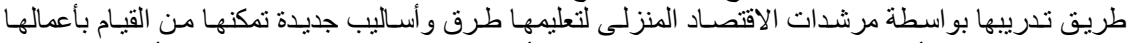

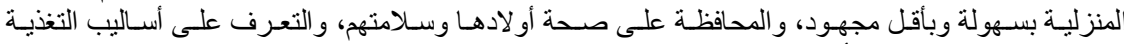

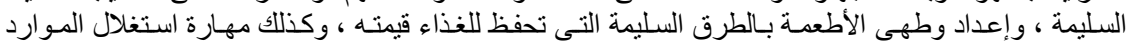

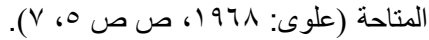

\section{الاستعراض المرجعى المنز}

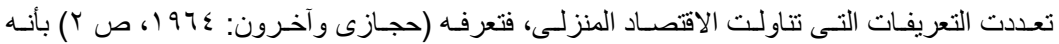

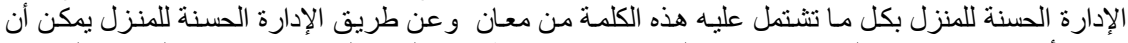

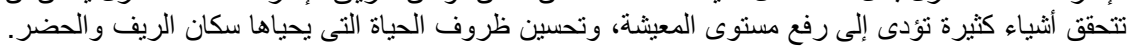

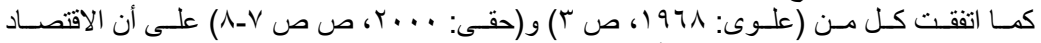

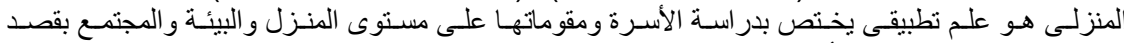
النهوض بها إلى حياة عائلية أفضل.

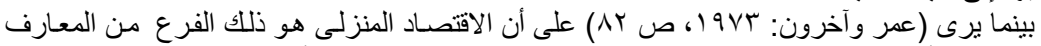

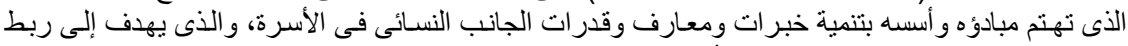

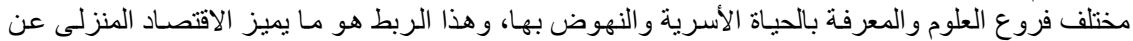
غيره من فروع الفنون والعلوم و المعرفة الأخرى. 


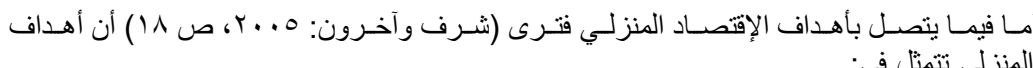

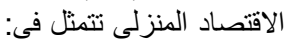

ا ـ تقوية وتطوير وارتقاء الحياة الأسرية بصفة عامـة والمر أة بصفة خاصسة من خلال تعليم الفرد سبل الحياة الأسرية

r- بـ كساعدة الأسر فى تحقيق أهدافها القريبة و البعيدة المدى.

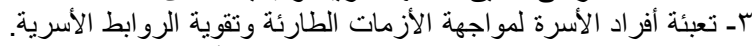

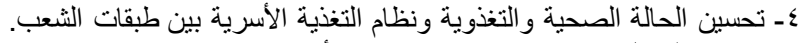

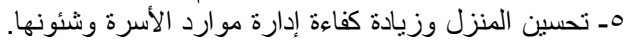

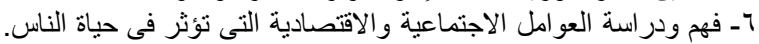

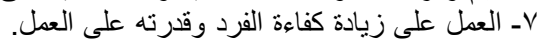

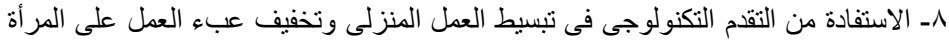

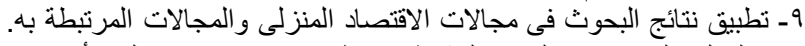

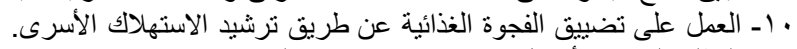
11 إ- التطلع إلى حياة أفضل في فلى حدود الإمكانيات المتاحة.

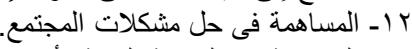

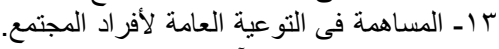

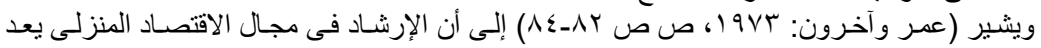

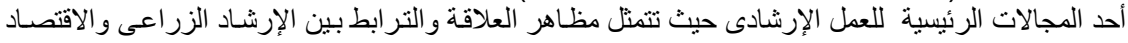

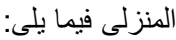

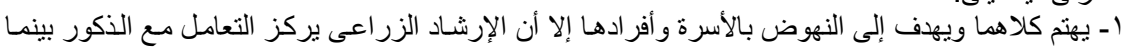

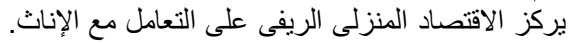

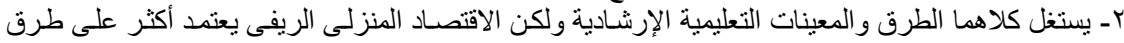

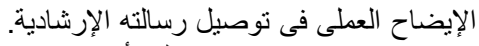

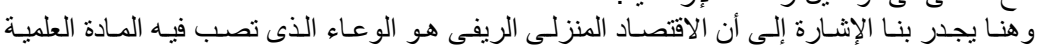

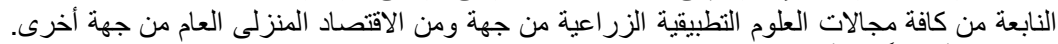

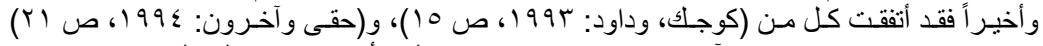

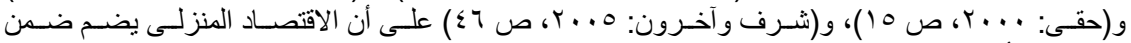

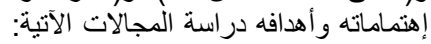
1 - 1 - 2 الغذاء و علوم الأطعمة.

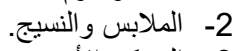
3- - المسكن الأسرى وأثناثه و أدو اته.

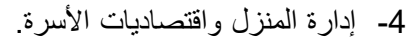
5- العلاقات الأسرية مع التركيز على نمو الطفل ورعايته.

\section{المشكلة البحثية البشية}

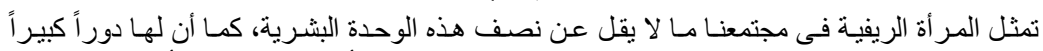

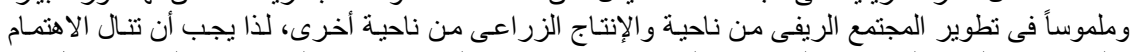

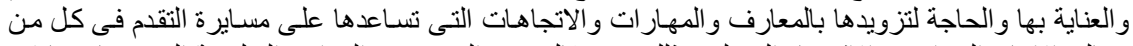

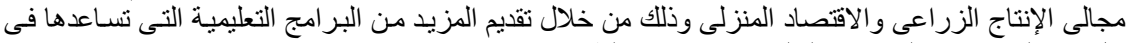

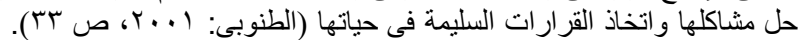

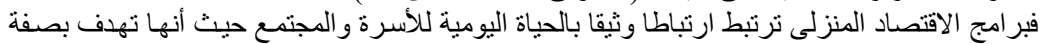

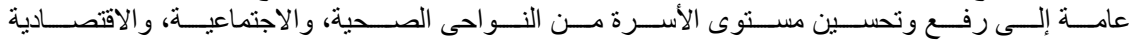

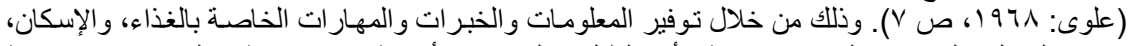

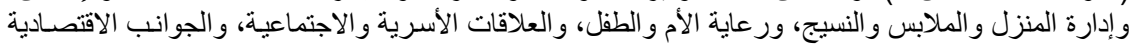

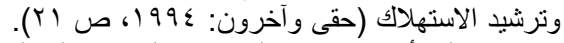

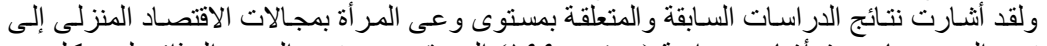

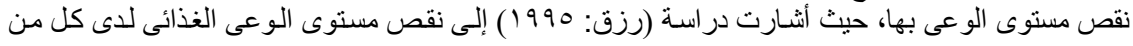




\section{El-Gamal, M.M.A. et al.}

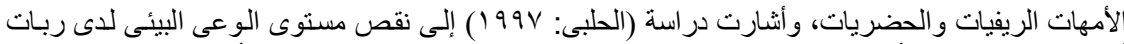

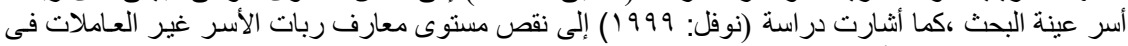

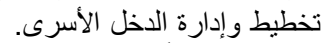

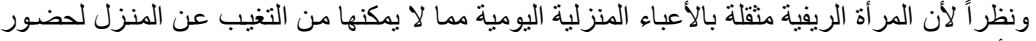

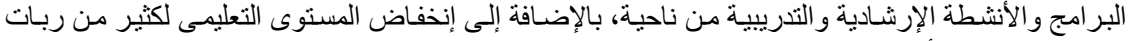

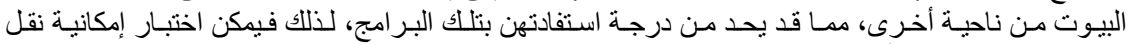

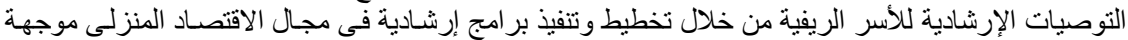

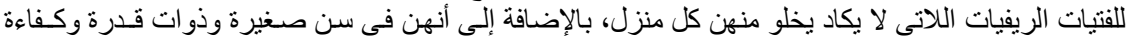

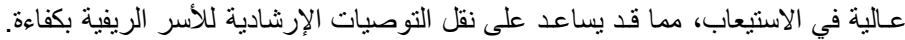

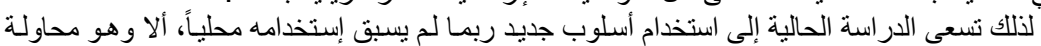

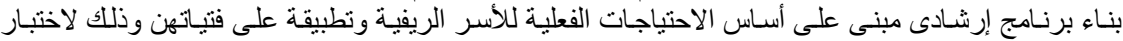

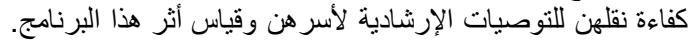

\section{أهداف الاراسة}

انطلاقاً من المشكلة البحثية قيد الدراسة، تسعى هذه الدر اسة إلى تحقيق الأهداف التالية:

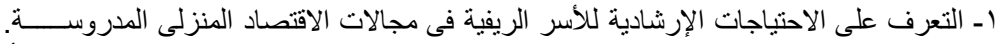

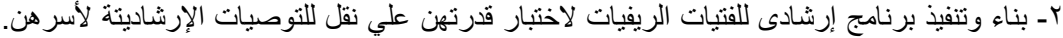

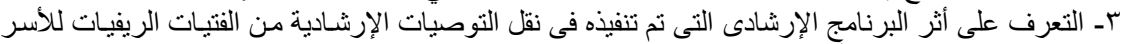
الريفية فى المجالات الأشند احتياجا.

\section{الطريقة البحثية}

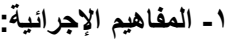

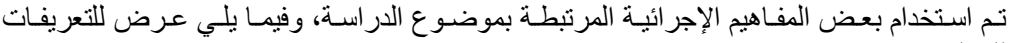

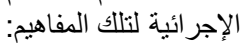

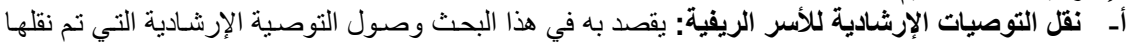

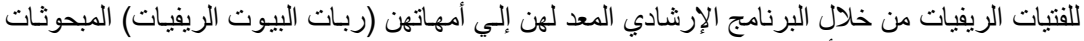

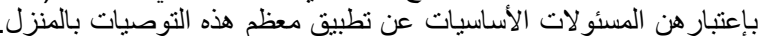

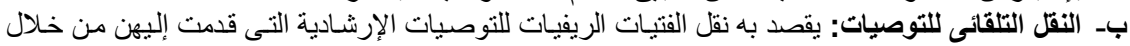

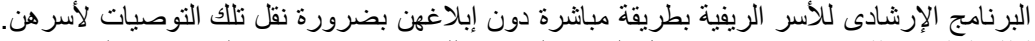

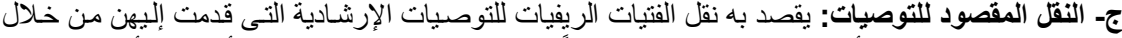

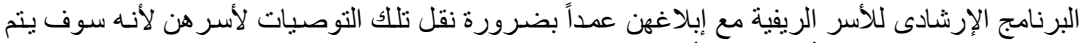

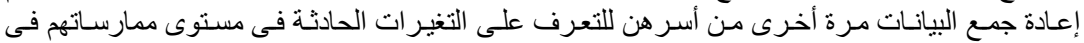

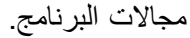

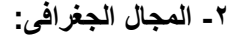

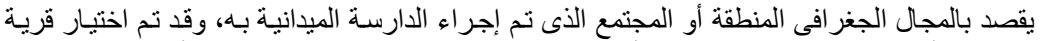

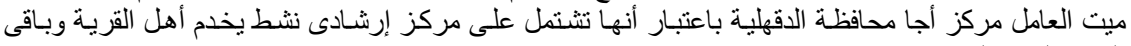

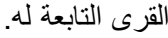

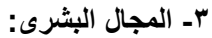

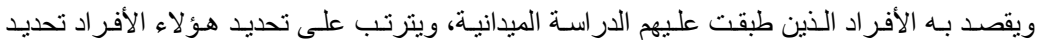

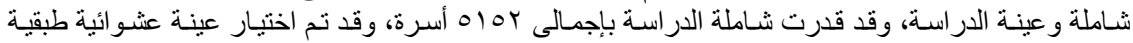

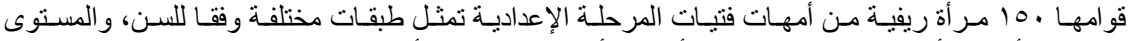

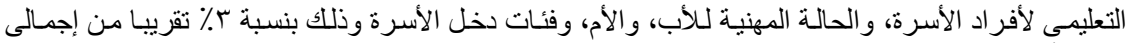

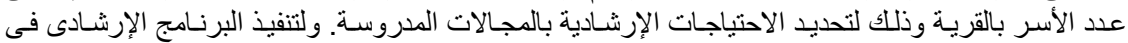

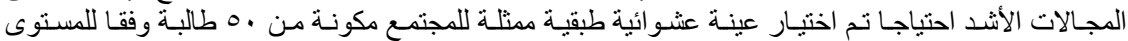

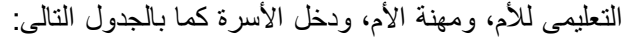

جدول رقم (1): توزيع أفراد عينة الفتيات الريفيات وفقا للمستوى التطليمى للأم ومهنة الأم ودخل الأسرة. 


\section{J. Agric. Sci. Mansoura Univ., 32 (4), April, 2007}

\begin{tabular}{|c|c|c|c|c|c|c|}
\hline \multirow{2}{*}{ المجموع } & \multicolumn{3}{|c|}{ فُئات دخل الأسرة } & \multicolumn{2}{|c|}{ مهنة الأم } & \multirow{2}{*}{ التعليمى المستوى } \\
\hline & $0 \ldots>\leq \ldots$ & $\varepsilon \ldots r \ldots$ & $r \ldots>r \ldots$ & لا تعمل & تعمل & \\
\hline ro & 0 & 0 & 0 & 0 & 0 & أمية \\
\hline ro & 0 & 0 & 0 & 0 & 0 & متعلمة \\
\hline 0 . & 1. & 1. & 1. & 1. & 1. & المجموع \\
\hline
\end{tabular}

ويقصد به الفترة الزمنية الفعلية التى تم خلالها جمع بيانات الدر اسـة الميدانية، حيث تم جمع البيانـات

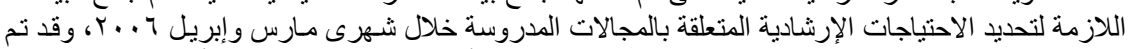

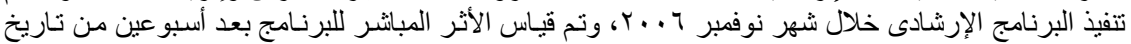

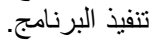

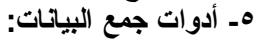

أ- إستمارة تحديد مستوى البات الاحتياج الإرشادى للمبحوثات في مجالات الاقتصاد المنزلى المدروسة:

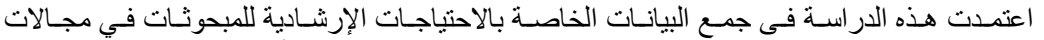

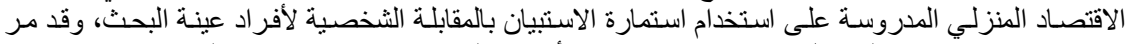

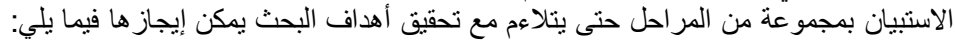

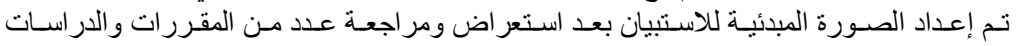

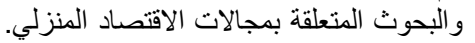

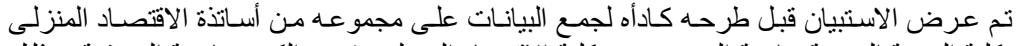

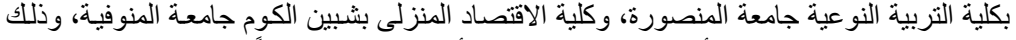

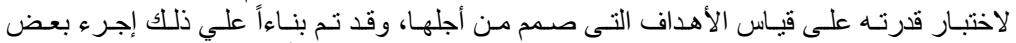

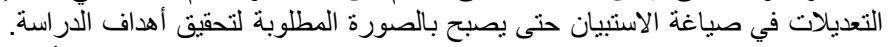

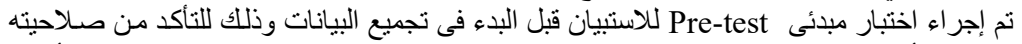

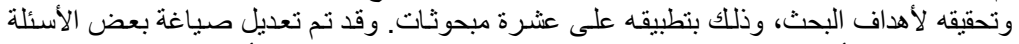

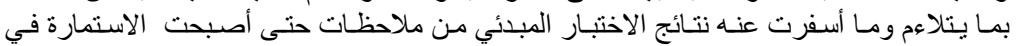

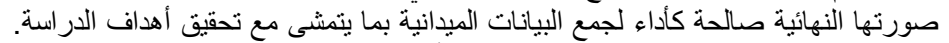

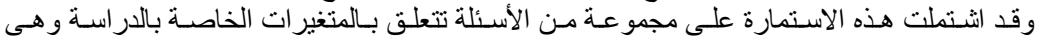

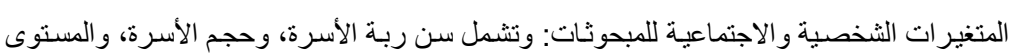

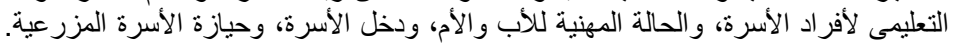

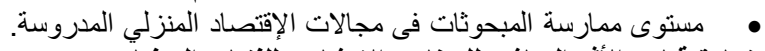

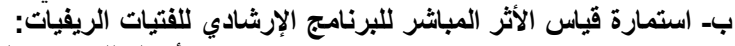

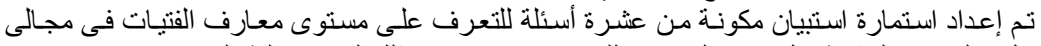

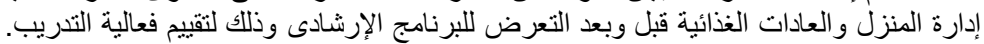

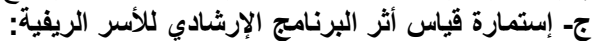

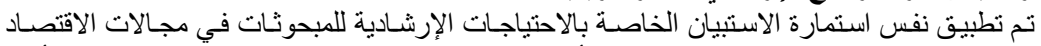

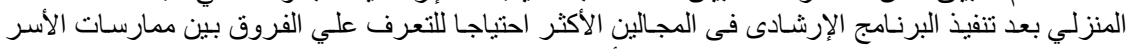

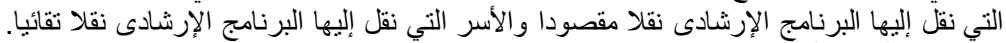

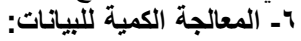

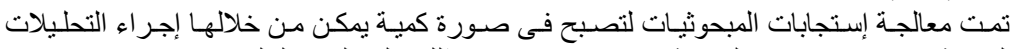

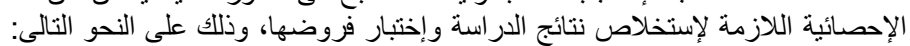

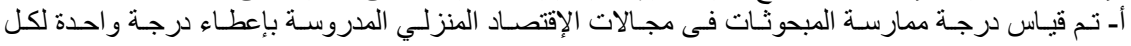
ممارسة صحيحة من ممارسات كل مجال مجال.

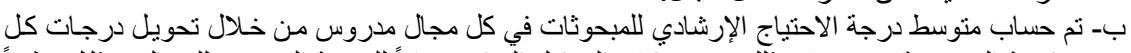

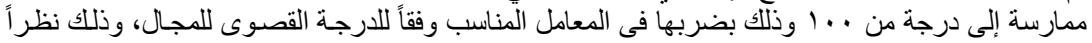

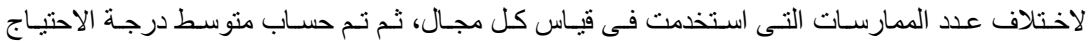

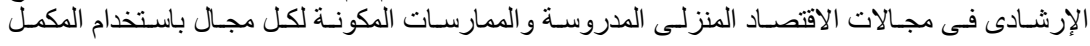


El-Gamal, M.M.A. et al.

$$
\begin{aligned}
& \text { المئوى وذلك بطرح قيم المتوسطات الحسبابية للممارسـات من . ․ 1، وبالتالى فإن الترتيب الأول يعطي }
\end{aligned}
$$

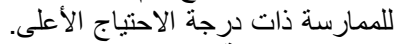

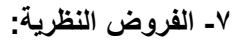

$$
\begin{aligned}
& \text { لتحقيق الهدف البحثى الثالث، تم صياغة الفروض النظرية التالية: }
\end{aligned}
$$

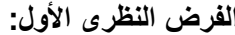

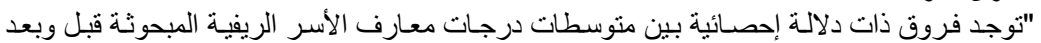

$$
\begin{aligned}
& \text { التعرض للبرنامج الإرشادى فيما يتصل بممارسات مجال إدارة المنزل و التصاديات دالتيات الأسرة". }
\end{aligned}
$$

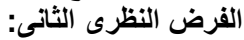

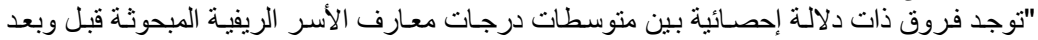

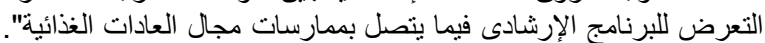

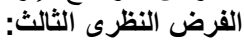

\section{النتائج ومناقشتها}

يعرض هذا الجزء النتائج التى أمكن الحصول عليها من الدراسة الميدانية وذلك على النئية النحو التالي:

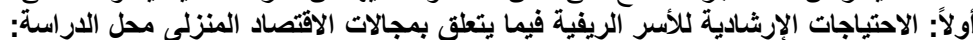

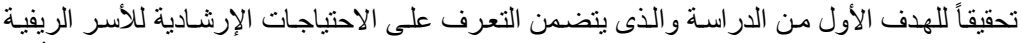

\begin{tabular}{|c|}
\hline متوسط درجة \\
\hline
\end{tabular}

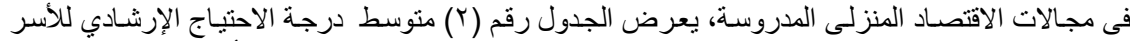

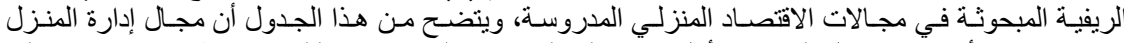

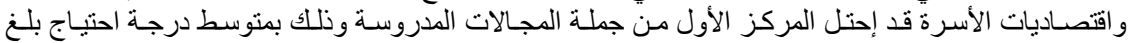

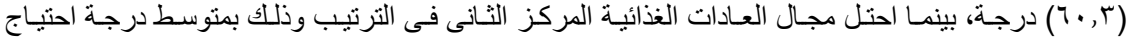

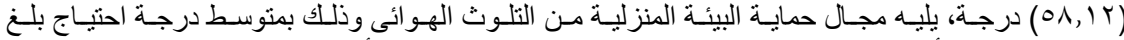

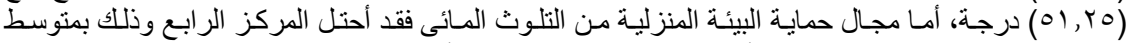

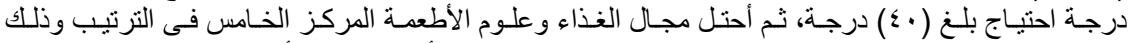

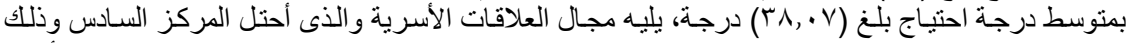

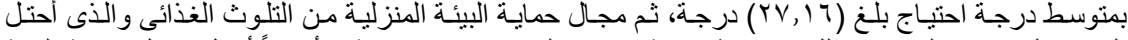

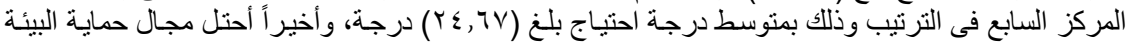

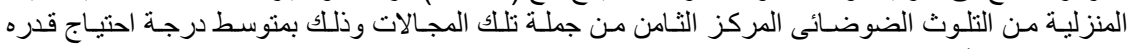

(IV, در درجة.

جدول رقم (r): توزيع مجالات الاقتصاد المنزلى وفقا لارجة الاحتياج الإرشادى للأسر الريفية المبحوثة. وفي الثرتيب

وفق درجة الحسبابي 
J. Agric. Sci. Mansoura Univ., 32 (4), April, 2007

\begin{tabular}{|c|c|c|c|}
\hline الاحتياج الإرشادى & الإرشادى & & \\
\hline 1 & $7 \cdot r$ & $r q, v$. & مجال إدارة المنزل و اقتصـاديات الأســــرة. \\
\hline 7 & $r V, 17$ & $V Y, \wedge \varepsilon$ & مجال العلاقات الأسريـــــــــة. \\
\hline V & $T \leqslant, T V$ & VO,ru & مجال حماية البيئة المنزلية من التلوث الغذائسى. \\
\hline$r$ & $01, Y_{0}$ & $\sum \Lambda, \vee_{0}$ & مجال حماية البيئة المنزلية من التلوث الهو ائسى. \\
\hline$\varepsilon$ & $\varepsilon \cdot, \cdot$ & 7.,. & مجال حماية البيئة المنزلية من التلوث المائسى. \\
\hline$\Lambda$ & $\mathrm{V}, \wedge \varepsilon$ & $\Lambda Y, 1\}$ & مجال حماية البيئة المنزلية من التلوث الضوضائى \\
\hline 0 & $r \Lambda, \cdot V$ & 71,94 & مجال الغذاء و علوم الأطعــــــــة. \\
\hline$r$ & $O \Lambda, 1 Y$ & $\S 1, \wedge \wedge$ & العــــــادات الغذائيــــــــة. \\
\hline
\end{tabular}

ثانياً: بناء وتنفيذ برنامج إرشادى للفتيات الريفيات لاختبار نقلهن للتوصيات الإرشادية لأسرهن:

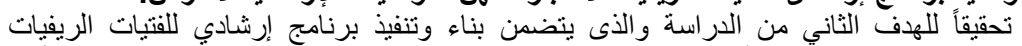

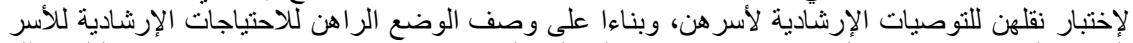

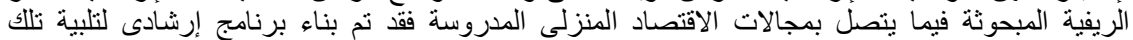

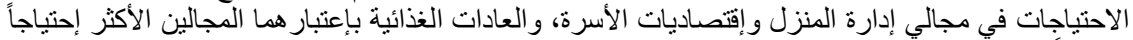

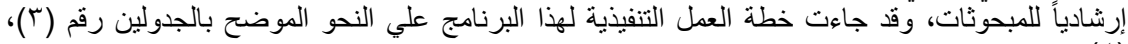

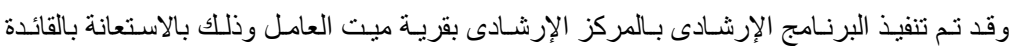

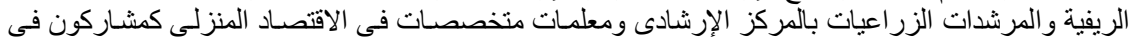

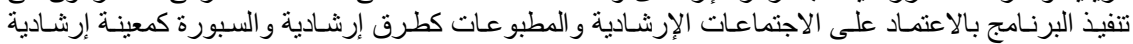

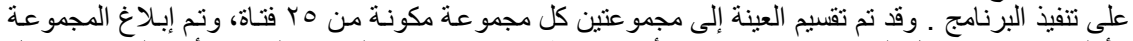

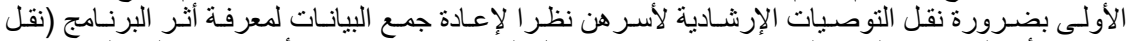

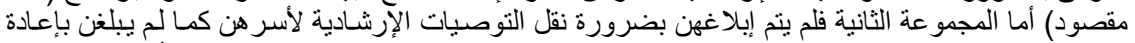

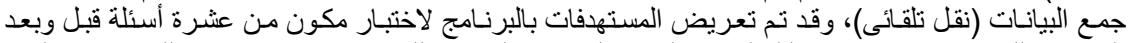

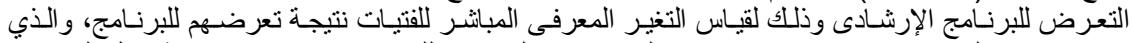

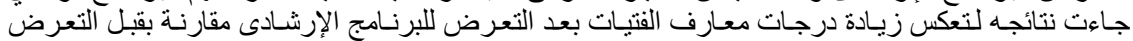
للبرنامج. 
El-Gamal, M.M.A. et al.

\begin{tabular}{|c|c|c|c|c|c|c|}
\hline تاريخ & مكان تنفيذ & القائمون بالتتفيذ & |الطرق والمعينات & المستهرفو & |الرسائل الإرشادية ( محتوى البرنامج ) & أهداف البرنامج | \\
\hline لمساء & الإرشادى المركز & 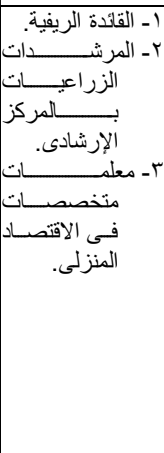 & 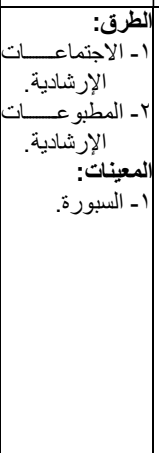 & (ألفراد الإعداديةة الفينة & 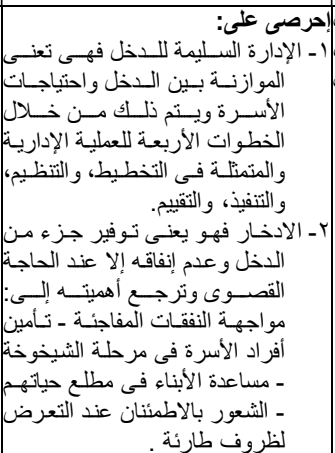 & 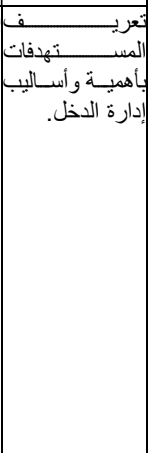 \\
\hline
\end{tabular}

جدول رقم (ع ): خطة العمل التنفيذية الخاصة بمجال العادات الغذائية.

\begin{tabular}{|c|c|c|c|c|c|c|}
\hline تاريخ & مكان تنفيذ & القائمون بالتنفيذ & الطرق الإرشاديةي & المستهوفي & الرسائل الإرشادية ( محتوى البرنامج ) & أهداف البرنامج \\
\hline يو يومي & الإرشادية ميث & 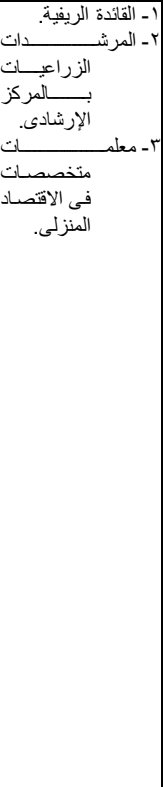 & 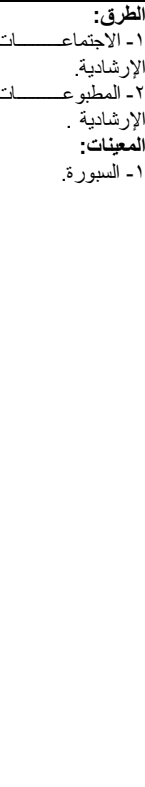 & الفتيات الريفيات & 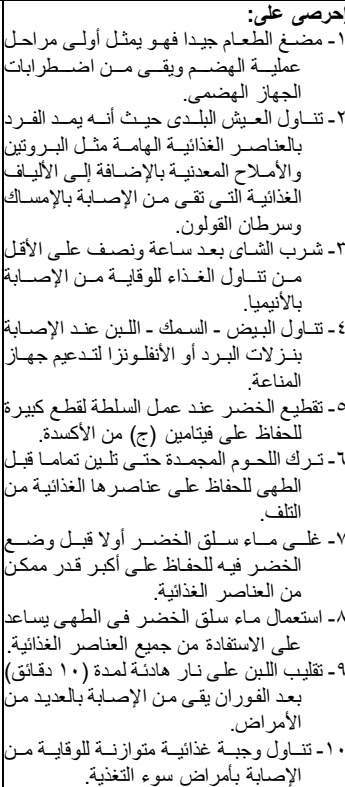 & 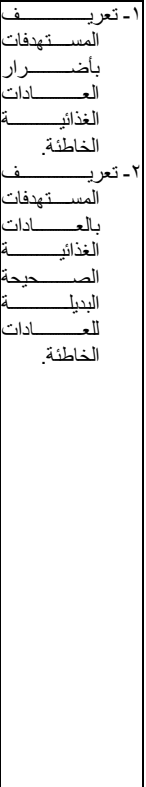 \\
\hline
\end{tabular}

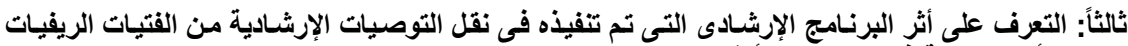
للأسر الريفية فى المجالات الأكثر احتياجا:

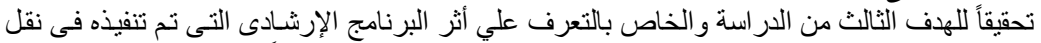

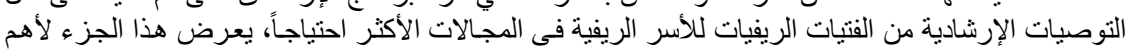
النتائج التي تم التوصل إليها في هذا التئ الصدد.

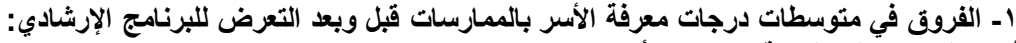
أ- مجال إدارة المنزل واقتصاديات الأسرة: 


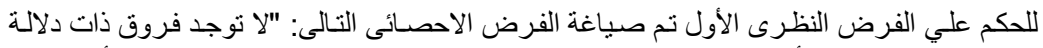

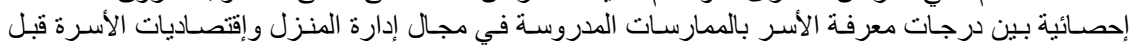

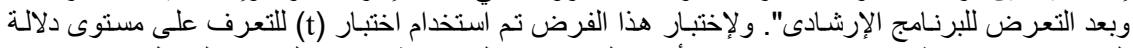

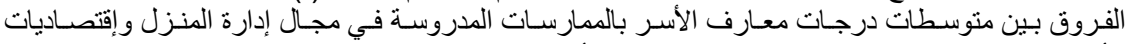

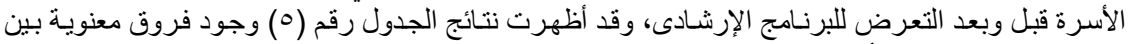

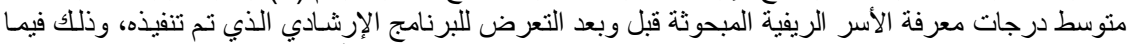

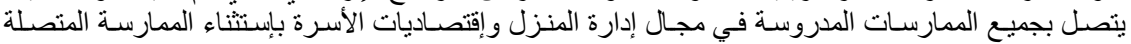

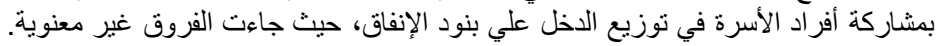

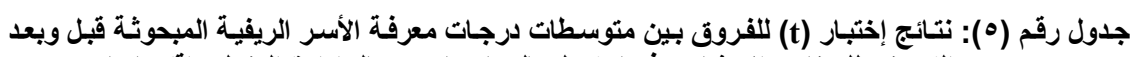

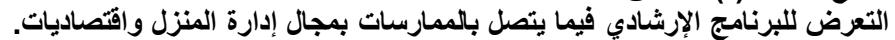

\begin{tabular}{|c|c|c|c|c|}
\hline \multirow[b]{2}{*}{ المعنوية } & \multirow[b]{2}{*}{ قيم } & \multicolumn{2}{|c|}{ المتوسطات } & \multirow[b]{2}{*}{ الممارسات المدروسة } \\
\hline & & البرنبامبج & البرنأمبلج & \\
\hline${ }^{* *} \cdot, \cdots$ & 11,1 & $r, \Lambda$ & $\cdot, 7$ & توزيع دخل الاسرة على بنود الإنفاق. \\
\hline$\cdot, 19$ & $1, \varepsilon r$ & r & $1, \varepsilon$ & مشّاركة أفراد الاسرة فى توزيع الدخل على بنود الإنفاق. \\
\hline$\pi \cdot, \cdot 1$ & T,, $\mathrm{\varepsilon}$ & t & 1 & اسباب توزيع الدخل على بنود الإنفاق. \\
\hline **,,$\cdots$ & $\vee, T \leqslant$ & $r, I r$ & $\cdot, 94$ & طرق الحفاظ على انتظام تنفيذ الميز انية المالية للاسرة. \\
\hline$\times *, \cdots$ & 0,94 & $r, 97$ & 1,1 & مدى ظهور ازمات مالبة في حباة الاسرة و او قات ذلك. \\
\hline$\because, \varepsilon$ & $T, T_{9}$ & 1 &., 00 & عمل حساب الإزمات بْادخار جزء من الاخخل. \\
\hline${ }^{* *}, \ldots$ & 7,07 & r, QY & $r, 1$ & مكافاة أو علاوة أو في أرباح. الزيسادة مسن الدخل فـى حالـة الحصسول على \\
\hline$\pi, \cdots$ & $\Gamma, .0$ & T & $\cdot, \lambda \varepsilon$ & مدى كفاية دخل الآسرة بسداد جميع اوجه الإنفاق. \\
\hline${ }^{* *} \cdot, \ldots$ & $\vee, \wedge q$ & 1,91 & 1,0 & الإخلِق المختلفة التى تلجـا إليها الاسرة فى حالهة حدوث عجز فى \\
\hline
\end{tabular}

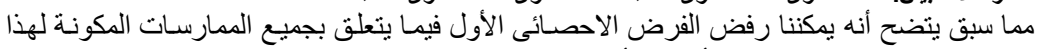

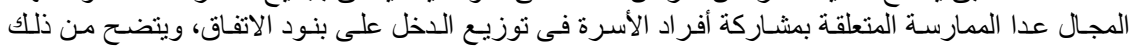

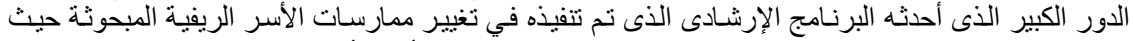

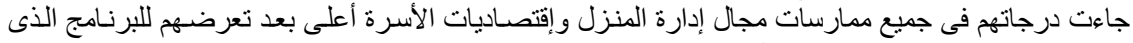
تم تنفيذه فيما عدا ممارسة ورئه واحدة فقط.

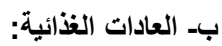

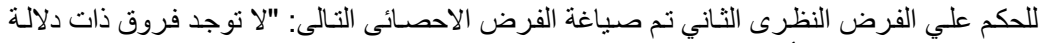

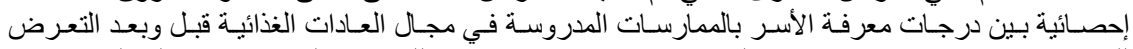

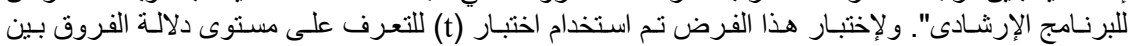

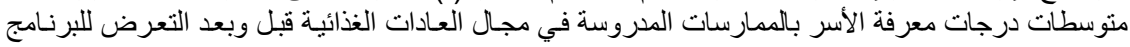

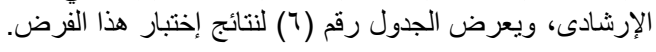

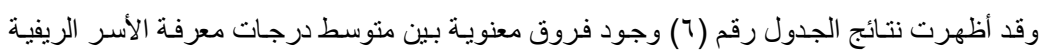

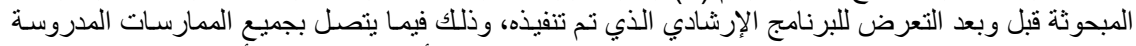

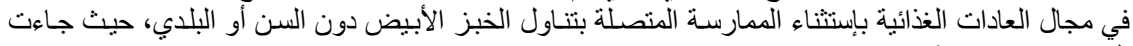
الفروق غير معنوية.

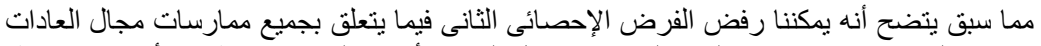

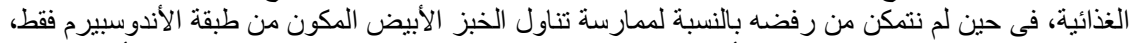

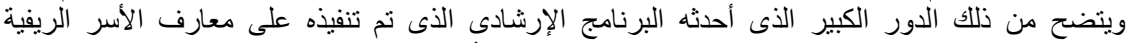

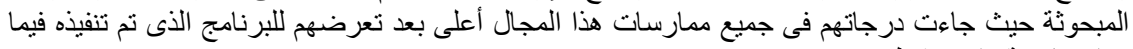
عدا ممارسة واحدة فقط.

جدول رقم (†): نتائج إختبار (t) للفروق بين متوسطات درجات معرفة الأسر الريفية المبحوثة قبل وبعد

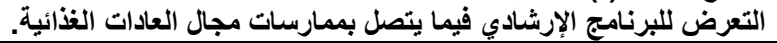

\begin{tabular}{|c|c|c|c|}
\hline مستوى & قِيم & المتوسطات & الممارسـات المدروسـة \\
\hline
\end{tabular}


El-Gamal, M.M.A. et al.

\begin{tabular}{|c|c|c|c|c|}
\hline المعنويا & $\mathbf{t}$ & 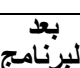 & | البرنَّمج & \\
\hline$\cdots{ }^{*}$ & $9, \mathrm{~V}$ & 1 & $\cdot, \pi$ & تناول الطعام بسر عة. \\
\hline$\cdot, \cdot \Delta \pi$ & $1,1 \times 7$ & r & $1,9 \leq$ & تناول الخبز الابيض دون السن او البلدى. \\
\hline$\because, \ldots$ & $\pi, 1 \mathrm{~V}$ & $T, \mathrm{~V}$ & $\cdot, \mathrm{VO}$ & شُرب النُّاى عقب الوجبات مباشُــرة. \\
\hline${ }^{*} \cdot, \cdot \mu_{\Lambda}$ & $r, 1 r$ & $\cdot, \xi$ & $\cdot, r \leq$ & 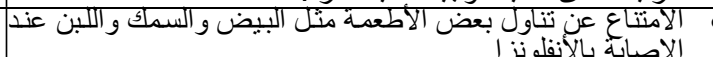 \\
\hline${ }^{x+} \cdot, \cdots 1$ & 11,9 & $\cdot, 9 \varepsilon$ & $\cdot, .01$ & تقطيع الخضروات ألسلطة لقطع صغيرة عند إعدادها. \\
\hline$\pi \cdot, \cdots$ & r, rq & $T, V$ & $1,7 \mathrm{~V}$ & وضتع اللحم او الدجاج المجمد في الماء لفك التُلج. \\
\hline${ }^{*} \cdot, \cdots$ & $7, Y$ & $\cdot, 97$ & $\cdot$, Or & غمر الخضار فى كمبة كبيرة من الماء تُم سلقه. \\
\hline$\bullet, \cdots$ & $r, \mathrm{~V}$ &., 97 & $\cdot, \cdot \mathrm{AV}$ & التخلص من الماء المستخدم في سلق الخضار \\
\hline$\because, \ldots$ & $\varepsilon, 7 Y$ & $\cdot, 9 \varepsilon$ & $\cdot, \pi$ & رفع اللبّن من على الموقد مبانُرة بمجرد فور انه. \\
\hline$\because, \cdots$ & $7, Y \leqslant$ & $\cdot, 9 \varepsilon$ & $\cdot, \leqslant 9$ & وضع اللحم في الماء لمدة نصف ساعة قبل الطهي. \\
\hline$\because, \cdots$ & $T, 1 \wedge$ & $\cdot, 9 \varepsilon$ & $\cdot, 1 \leqslant$ & تقديم البطاطس مع الارز او الخبز فقط في وجبة غذائية واحدة. \\
\hline
\end{tabular}

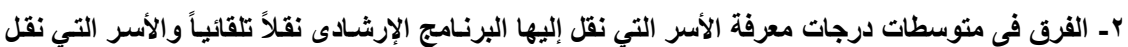

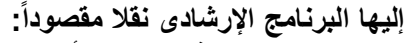
أ- مجال إدارة المنزل والإنتصاديات الأسرة:

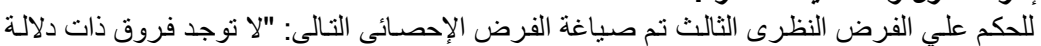

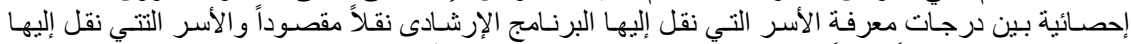

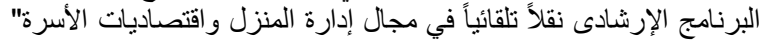

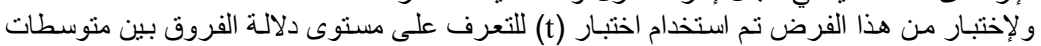

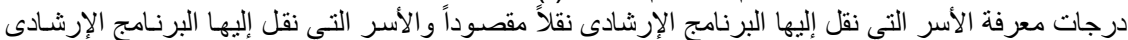

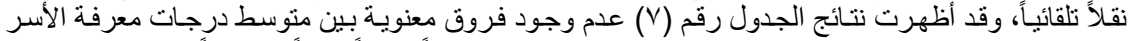

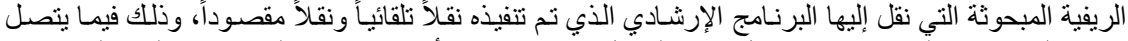

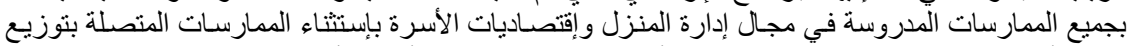

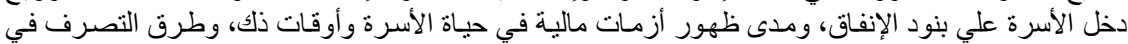

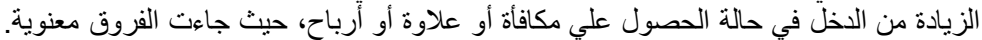

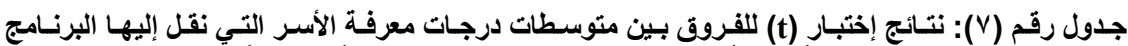

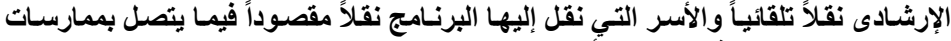

لمجال إدارة المنزل واقتصاديات الأسرة.

\begin{tabular}{|c|c|c|c|c|}
\hline \multirow{2}{*}{ المعنوية } & \multirow{2}{*}{ قيم } & \multicolumn{2}{|c|}{ المتوسطات } & \multirow[b]{2}{*}{ 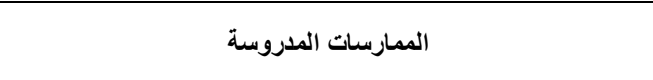 } \\
\hline & & نقل تلقائي & نقل مقصود & \\
\hline **** & $\varepsilon, 91$ & Y, & $r, r$ & توزيع دخل الاسرة على بنود الإنفاق. \\
\hline • & 1,11 & 1,9 & $r, r$ & مشاركة أفراد الاسرة فى توزيع الاخل على بنود الإنفاق. \\
\hline$\cdot, Y_{1}$ & $\cdot, 10$ & $1, \varepsilon$ & 1,0 & أسباب توزيع الدخل على بنود الإنفاق. \\
\hline$\cdot, Y Y \cdot$ & $\cdot, Y V$ & $1,9 r$ & 1,90 & طرق الحفاظ على انتظام تتفيذ الميز انية المالية للاسرة. \\
\hline$\pi$ & $\varepsilon, O Y$ & $r, 1$ & 0 & مدى ظهور أزمات مالية فى حياة الاسرة وأوقات ذلك. \\
\hline- & - & 1 & T & عمل حساب الاززمات بادخار جزء من اللخل. . \\
\hline${ }^{* *} \cdot, \cdot 1$ & $1, r$. & $r, 97$ & $r, \wedge T$ & أو علاوة ألتصر أرباح. فى الزيادة من الدخل فى حالة الحصول على مكافاةً \\
\hline - & - & 1 & 1 & مدى كفاية دخل الاسرة بسداد جميع أوجه الإنفاق. \\
\hline$\cdot,+7$ & 1 & 1,9 & r & الطخل. الطرق المختلفة التى تلجـا إليهـا الاسرة فى حالـة حدوث عجز فى \\
\hline
\end{tabular}

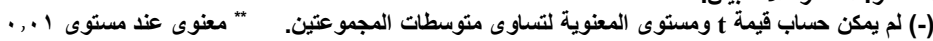

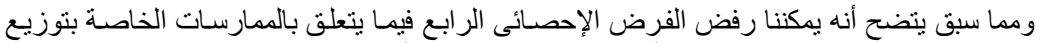

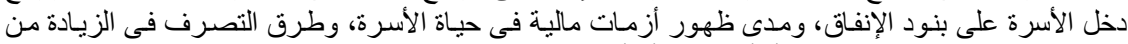

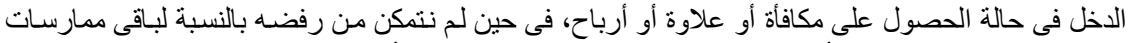

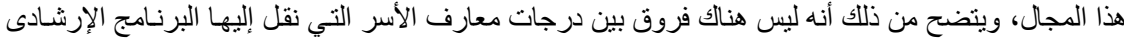


نقـلاً تلقائياً وتلك التي نقل إليها البرنامج نقلاً مقصوداً بإستثناء ثناث ممارسـات فقط من إجمالى ممارسـات هذا

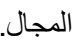

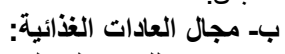

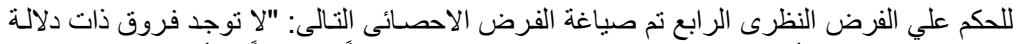

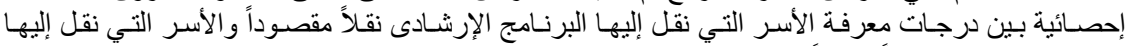

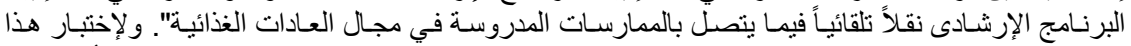

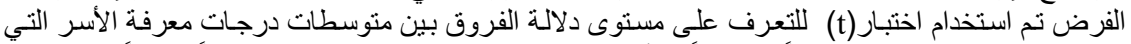

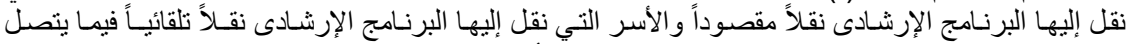

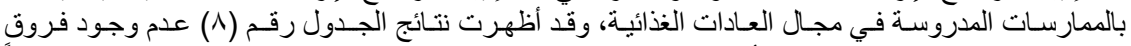

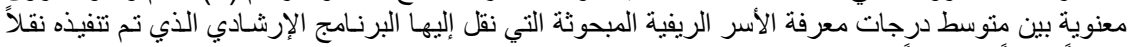

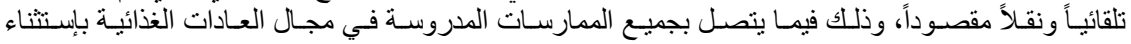

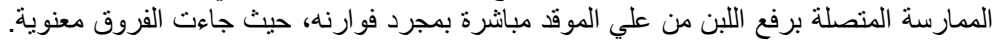

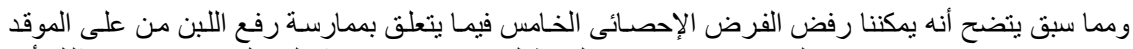

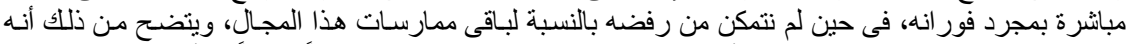

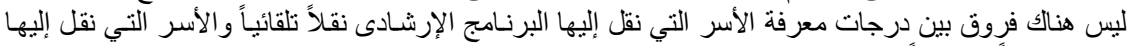

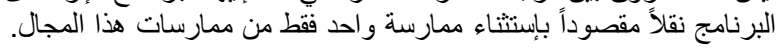

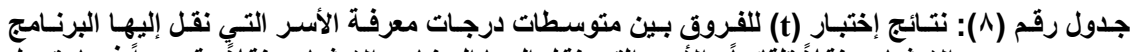

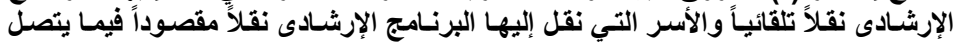

بالممارسات المدروسة في مجال الألئر التعادات الغذائية.

\begin{tabular}{|c|c|c|c|c|}
\hline \multirow[b]{2}{*}{ المعنوية } & \multirow[b]{2}{*}{ قيم } & \multicolumn{2}{|c|}{ المتوسطات } & \multirow[b]{2}{*}{ الممارسات المدروسة } \\
\hline & & البرنامج & البرنَّلَ & \\
\hline- & - & 1 & 1 & تتاول الطعام بسر عة. \\
\hline- & - & r & r & تتاول الخبز الابيض دون السن او البلدى. \\
\hline$\cdot, Y \varepsilon$ & 1, 0 & $T, 7$ & $T, \varepsilon 0$ & شرب الشّى عقب الوجبات مباشرة. \\
\hline • & $\cdot, \leq 0$ & 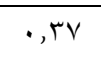 & $\cdot, \varepsilon \Gamma$ & 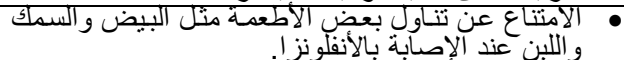 \\
\hline$\cdot, T V$ & ,,$\leqslant 0$ & $\cdot, 97$ & $\cdot, 90$ & تقطيع خضروات السلطة لقطع صغيرة عند إعدادها. \\
\hline$\cdot, 1 \varepsilon$. & $\cdot, 70$ & T,77 & $T, V \lambda$ & وضنع اللحم او اللاجاج المجمد في الماء لفك ألتلج. \\
\hline$\cdot, \wedge r$ & $\cdot, 11$ & $\cdot, 97$ & $\cdot, 90$ & غمر الخضار فى كمية كبيرة من الماء ثم سلقه. \\
\hline$\cdot, \varepsilon \varepsilon$. & $1, T M$ & $1, r$ & $\cdot, \mathrm{V}$ & التخلص من الماء المستخدم في سلق الخضار. . \\
\hline **,,$\cdots$ & 1,77 & $\cdot, \wedge \wedge$ & 1 & رفع اللبن من على الموقد مباشُرة بمجرد فورانه. \\
\hline$\cdot, \mathrm{TV}$ & $\cdot, \leqslant 0$ & $\cdot, 97$ & $\cdot, 90$ & وضع اللحم في الماء لمدة نصف ساعة فبل الطهى. \\
\hline 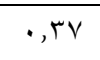 & $\cdot, \leqslant 0$ & $\cdot, 94$ & $\cdot, 90$ & تقديمة البطاطس مع الأرز أو الخبز فقط فى وجبـة غذائية \\
\hline
\end{tabular}

\section{توصيات البحث}

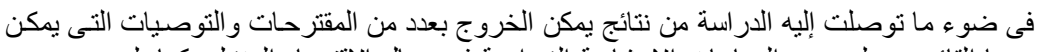

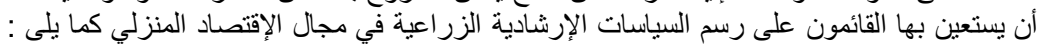

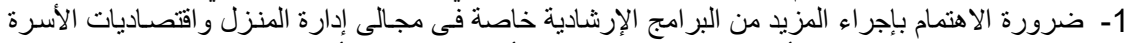

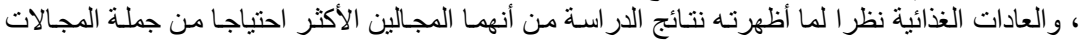

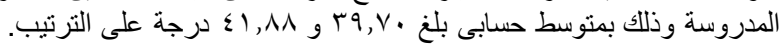

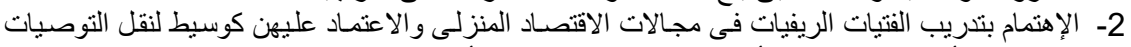

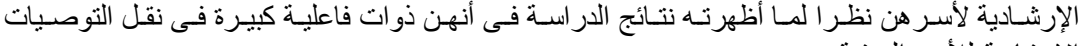
الإرشادية للأسر الريفية. 


\section{El-Gamal, M.M.A. et al.}

3- إزاء ما تبين من فاعلية البرنامج الإرشادى الذى تم تنفيذه فى تغيير المستوى المعرفى للفتبات الريفيات

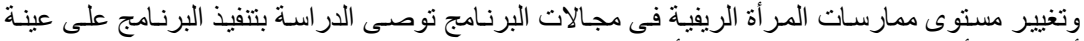

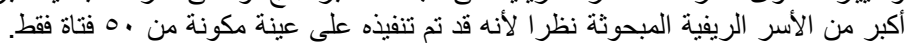

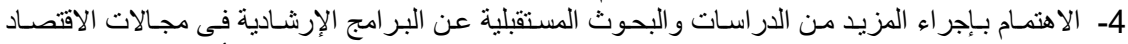

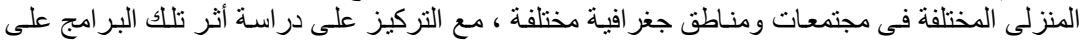
مستهوفيها فى مختلف النواحى الاجتماعية والاقتصادية والبيئية.

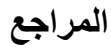

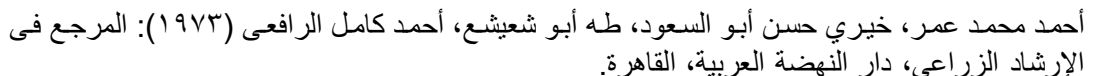

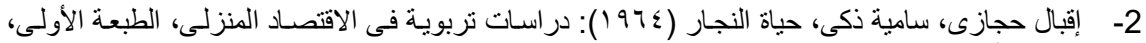
مكتبة الأنجلو المصرية، القاهرة.

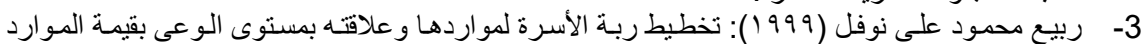

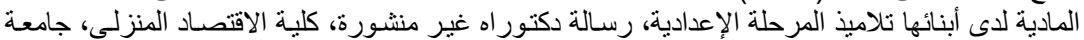

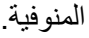

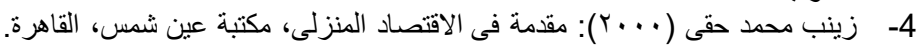

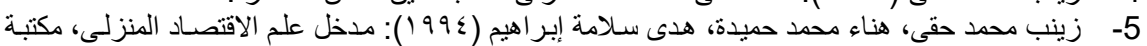
عين شمس، القاهرة.

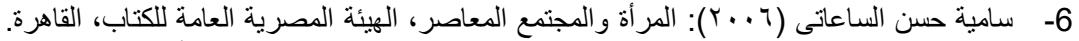

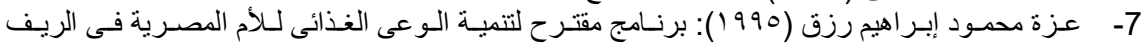

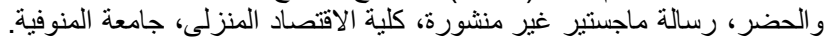

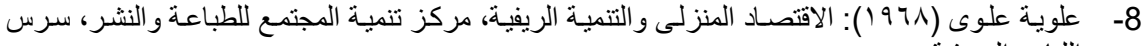

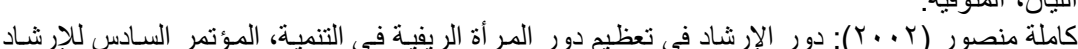

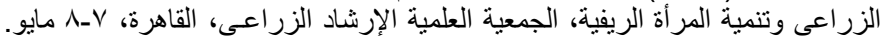

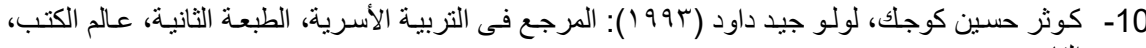

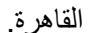

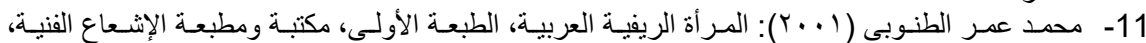
الإسكندرية.

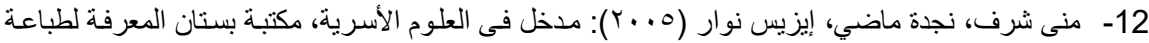
ونشر وتوزيع الكتاب، ألقاهرة.

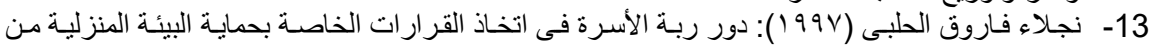
التلوث، رسالة ماجستير غير منشورة، كلية الاقتصاد المنزلى، جامعة المنوفية. 


\title{
A STUDY OF RURL GIRLS EXTENSION PROGRAMS EFFECT IN TRANSFERRING EXTENSION RECOMMENDATIONS TO RURAL FAMILIES IN THE FIELD OF HOME ECONOMICS "A FIELD STUDY IN RURAL DAKAHLIA GOVERNORATE". EI-Gamal, M.M.A.; M.A.M. Abd El-Magieed and Zozo F.E. Sallam Agricultural Extension and Rural Society Dept., Faculty of Agriculture, University of Mansoura.
}

\begin{abstract}
This study aimed at testing the effect of extension programs delivered to rural girls in transferring extension recommendations to rural families in the field of home economics. The study was carried out in Aga District at Dakahlia Governorate. Meet EL-Amel village was selected from this district because it has a powerful agricultural extension center. A stratified random sample of 150 rural women was selected for studying the extension needs in the studied home economics fields. The sample represented $3 \%$ of rural families in the village. Also, a stratified random sample of 50 basic education female students was selected for conducting the extension program in the highly needed fields of home economics. Data were collected by using personal interview questionnaire from sample rural women during the period from March to April 2006. The extension program of basic education female students was carried out during the period from 10-11 November 2006. The direct effects of the program were measured two weeks after carrying out the program. Frequencies and t-test were used to analyze data statistically.

The study reached a number of important findings. These findings indicated that the fields of home management and family economics, and nutritional habits came as the highly needed fields among the studied home economics fields. Moreover, the findings revealed that there are significant differences between sample rural women before and after conducting the extension program regarding the averages of practice degrees related to home management and family economics, and nutritional habits. Finally, the findings indicated that there are no significant differences between sample rural families who received the content of extension program intentionally and spontaneously concerning the averages of practice degrees related to home management and family economics, and nutritional habits.
\end{abstract}

